\title{
Understanding Crowd Dynamics in Processions during Mass Religious Gatherings A case study of Shahi Snan in Kumbh Mela
}

\author{
H Gayathri ${ }^{1}$, Siddhartha Gulhare ${ }^{2}$, Ashish Verma ${ }^{3 *}$ \\ ${ }^{1}$ Research Scholar, ${ }^{2}$ ex- Project Associate \\ Department of Civil Engineering, \\ Indian Institute of Science (IISc), Bangalore 560012, India \\ gayuhariharan@gmail.com; siddharthagulhare89@gmail.com \\ ${ }^{3}$ Associate professor, Department of Civil Engineering and \\ Robert Bosch Centre for Cyber Physical Systems, \\ Indian Institute of Science (IISc), \\ Bangalore 560012, India \\ ashishv@iisc.ac.in \\ *(Corresponding Author)
}

\begin{abstract}
Large people gatherings in public places exhibit crowd dynamics that are quite complex. Such mass events with high densities are fraught with potentially serious consequences if not understood and managed properly. In mass religious processions, the participants in the crowd are emotional and mostly impulsive and often crowd disasters occur due to the behaviour of the crowd. These events have great potential to cause safety hazards to the people. This paper attempts to narrate the typical situations of crowd dynamics observed in the Kumbh Mela procession-2016 and to describe the characteristics of the crowd that have not been reported in literature so far but have a significant impact on the crowd. Extreme crowd pressures resulting in individual loss control due to psychological and physiological factors, heterogeneity in the crowd, group behaviour and their induced competitiveness, unexpected behaviour exhibited due to the motivation behind participating in such procession makes it a typical crowd concentrated event to study the potentially critical dynamics of crowd. Physical and psychological forces acting on the people and their resulting dynamics of crowd in the Kumbh Mela procession 2016 lead to serpentine behaviour, which can possibly lead to crowd crushes, or any such crowd risk situations. Therefore, the characteristics of crowd participating in the Kumbh Mela procession have to be clearly understood so that it helps in better planning and well-organized movement patterns.
\end{abstract}

Keywords: Group Behaviour; Pedestrian; Mass religious gatherings; Crowd risk; India

\section{Introduction}

Crowds are extremely complex systems.Large number of people gather in places like temples, mosques, shopping malls, bus and train stations, etc. and in places of particular attractions or events that take place like sports events, concerts, theatres, religious gatherings, carnivals (melas), processions, etc. Each crowd is a unique phenomenon and the nature of the crowd is difficult to generalize. In a mass procession, the participants in the crowd are emotional and mostly impulsive and often crowd disasters occur due to the behaviour of the crowd. At high densities, there is the potential for overcrowding which could result in personal injuries due to the role of situational influences. Therefore understanding the complex crowd dynamics in such mass processions is a crucial step towards eliminating the crowd disasters.

There have been several studies on how crowd disasters happen. The inadvertent body contacts between pedestrians induce high forces that propagate along the direction in which they exert (Ma et al, 2013, Kolli, 2014, Dave, 2014, Fruin, 1993, Helbing et al, 2007, Moussaid et al, 2011). In addition, many studies have revealed that people mostly come in groups at leisure places like shopping malls, football events, etc. (Coleman \& James 1961, Aveni 1977, Moussaid et al 2010 and Cheng \&Yarlagadda 2014). Pedestrian groups generally develop patterns (Strogatz et al, 2005, Moussaid et al, 2010, Miguel, 2015) and thereby reduces the travel time (Koster et al, 2011). In mass religious processions like Kumbh Mela, people participate with a common objective of taking a dip in ariver believing it . These goal-oriented 
crowds are concerned about the safety of their affiliated members and so mostly consists of groups. A questionnaire survey conducted by the authors in Kumbh Mela-2016 revealed that $95 \%$ of surveyed people came in groups. Many researchers also study the competitiveness between members in crowd (Kretz, 2007, Fruin JJ, 1993, Darwin, 1871). When large number of people gather and move in a constrained environment with common goals, they find themselves in dangerous conditions.

Many processions that happened across the world have resulted in life-threatening disasters due to the unexpected behaviour of the crowd. Some of the examples of crowd disasters in processions are funeral procession in Moscow (Pozner and Vladimir, 1990), crowd crush in Shia procession ("Reaction to Iraq stampede", BBC News, 31 August 2005), crowd crush in Varanasi religious procession ("Varanasi: Deadly stampede at Hindu procession", CNN International Edition, 16 October 2016) and crowd crush in Kumbh Mela procession ("The worst stampede in Allahabad in 1954, killing 800", The Guardian, 28 August 2003). Crowd crush often occur when a majority of people in an event with mass gathering tries to move towards a central attraction (Dave, 2014). These crowd concentrated events have a great potential to cause safety hazards to the people. Therefore finding a sensible description of the various aspects of crowd behaviour and its characteristics in mass processions with high degree of complexities involved is an important issue. Nevertheless, this has to be understood to provide opportunities to change crowd dynamics and to avoid any adverse effects in future events.

\section{Qualitative analysis- A case study of Shahi Snan}

Shahi Snan (royal holy bath) is the major event of Kumbh Mela festival, which takes place on predetermined dates. During this time, river Kshipra is the point of central attraction, as it is believed to wash the sins of all previous births. Before taking bath, there goes a long procession of saints and their disciples from various ascetic orders. Several saints come in the procession sitting on horses and decorated chariots drawn by their disciples. Uniquely, Kumbh Mela is one mass religious event that gives opportunity to study all the typical situations of crowd dynamics observed in such events. The scenario of crowd is very different. (Gayathri et al, 2017). This work attempts to study the situations in Kumbh Mela procession 2016 that may lead to crowd disasters in future events and recommendations/solutions to prevent such disasters in future are suggested. In Kumbh Mela-2016 Shahi Snan, as per the government, 2.5 million people visited the city (Economic Times, May 09, 2016). Fig. 1 shows the GPS trace of a participant during shahi snan procession in Kumbh Mela-2016.

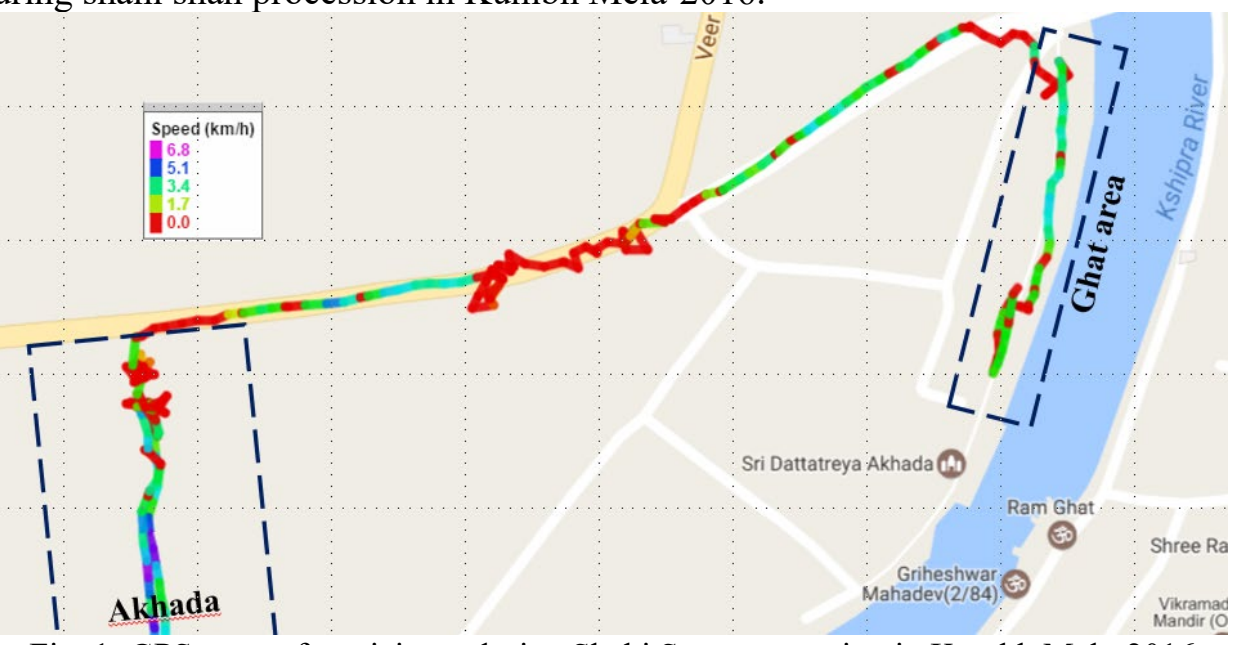

Fig. 1: GPS trace of participant during Shahi Snan procession in Kumbh Mela-2016

The shahi snan procession-2016 is a well-planned event. The saints travel in their tractor-drawn chariot and their respective disciples would follow them. It was planned in such a way that the tractor-drawn chariots would control the crowd situation at macroscopic level to some extent. Also, since people spend time in bathing in the ghat area, the inflow is to be curtailed from time to time in such a way that the rate of people entering the ghat is less than or equal to the rate at which they are exiting the ghat. 
In order to avoid overcrowding in the ghat area, the chariots will have to wait at the ghat entrance and will be allowed to enter at certain intervals. The planned scenario for the procession is depicted in Fig. 2.

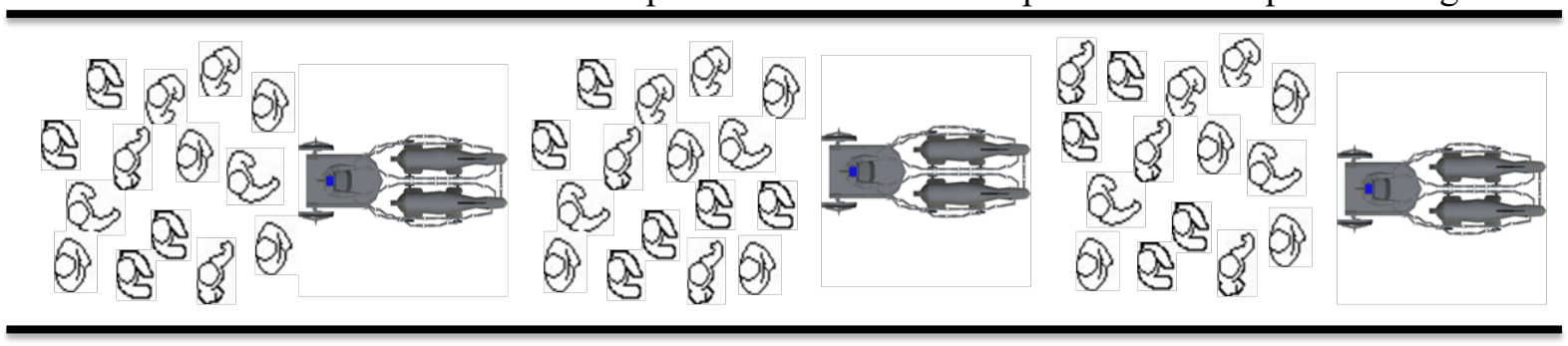

Fig. 2: Representation of planned scenario for the procession

\section{Observed crowd characteristics in Kumbh Mela procession-2016}

The actual situation observed in Kumbh Mela procession-2016 was different from the planned scenario. On the day of procession, saints and disciples from 13 akhadas participated in the procession. The pilgrims gathered in their respective saints' camp from late evening. The procession started as planned but with a delay of 2 hours due to which the pilgrims became impatient. The delay is believed to be due to some rituals in some akhadas. A team including authors joined and observed the procession. The team members walked alongside the procession carrying GPS devices and head mount camera and visually observed the impact of the event on the crowd as they moved forward towards the ghat for holy dip. Snapshots of Shahi Snan procession in Kumbh Mela 2016 is shown in Fig. 3.
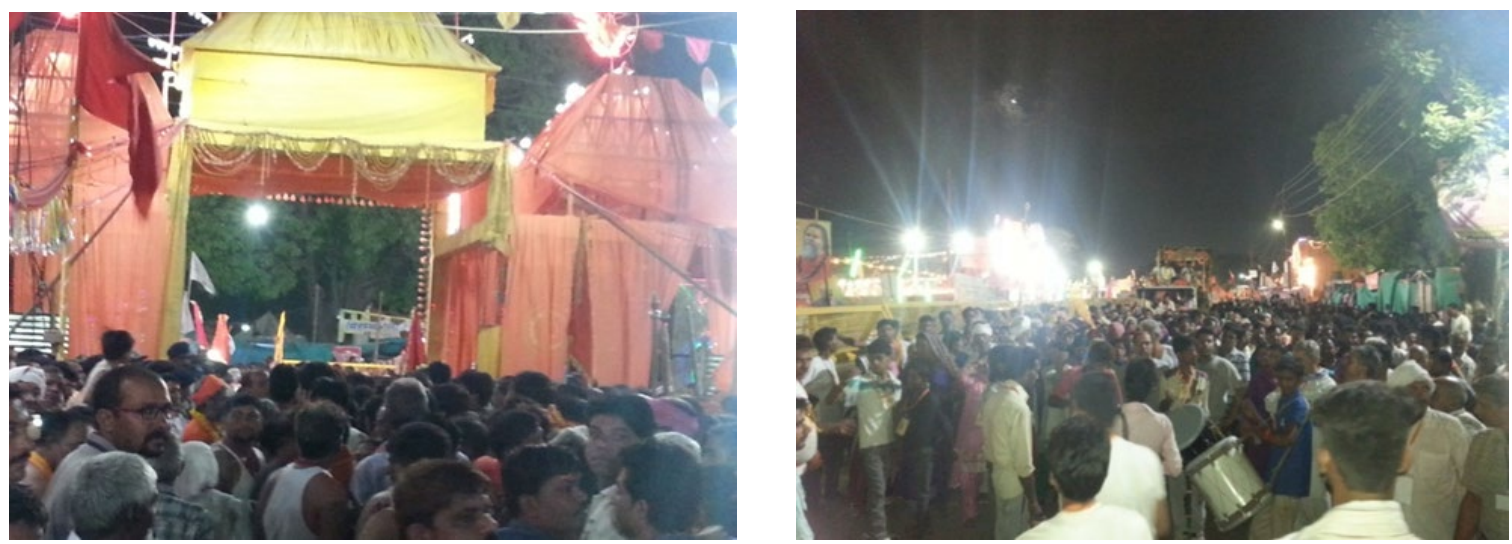

Fig. 3: Snapshots of Shahi Snan procession

As the procession began, the camps started leaving the akhadas one after another. As the procession started with a delay, people became impatient and tried to exit their camps and join the procession with high desirable speed. Near the front gate of one of the camps (Niranjani Akhara) people recognized that the other camp people were trying to overtake them and so they tried to increase their speed rapidly. The front gate of the camp was narrow and this created a bottleneck effect near the gate which resulted in pushing. People at the rear end, unaware of the situation at the front, kept moving while those in the front experienced severe distress. The smaller densities at the rear end allowed people to move freely but those at the front were immobile and under extreme pressure. Therefore, people started behaving aggressively as their urge to move forward as quickly as possible towards the ghat to take holy dip increased and the spiritual gain that they derive from the rare occasion being with their saints, made them feel like they are privileged.

As the crowd progressed towards the destination, the density became high. Some people who don't belong to the akhada and do not hold a permission to participate in the procession tried to enter the procession and overtake the pilgrims and saints from sides to reach the ghat to take holy dip. Therefore, the pilgrims started to take the holy dip as a 'chance to gain' and more likely as a competition to reach the ghat as quick as possible. Therefore, a cooperative behaviour, which is very important in mass gatherings like Kumbh procession to avoid crowd risk situations, was missing. This competitiveness of crowd only 
added instability in the crowd leading to decrease in flow rate and resulted in altruism among people in groups. They had the desire to overtake others and move forward and so tried to find out gaps in the crowd to sneak through. This added complexity to the crowd. The actual situation witnessed in the Kumbh Mela procession 2016 is depicted in fig. 4. Pedestrians in red indicates serpentine group and blue inindicates people who try to overtake along the sides.

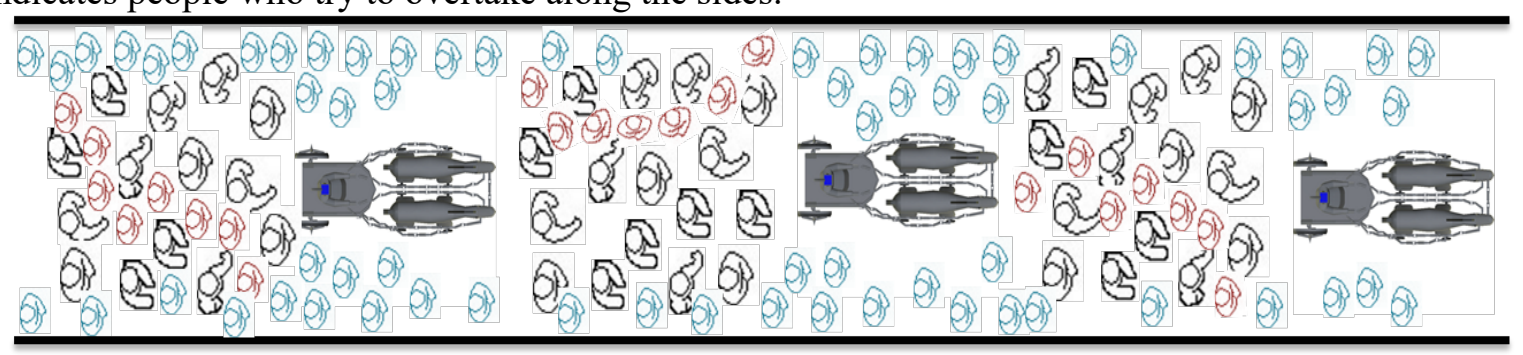

Fig. 4: Representation of actual situation observed in the procession

Some people followed their saints and moved along the crowd but some people tried to sneak through the crowd to reach the ghat as early as possible. They overtook others including the saints' tractor drawn chariots to reach the ghat entrance. Security personnel using temporary barricades stopped people $100 \mathrm{~m}$ before the ghat entrance and allowed them to enter the ghat at suitable intervals to avoid overcrowding at the ghat section. The saints were allowed to enter first and the pilgrims were stopped near the ghat entrance and allowed at regular intervals. The people at the rear end, unaware of the situation ahead, kept moving forward even when the flow is stopped and this increased the density at the near end. Some impatient pedestrians tried to overtake the crowd and reach the ghat entrance. Also people from sides tried to merge with the crowd and this lead to high densities near the entrance resulting in stop and go waves propagating in the opposite direction. Interaction of these factors lead to a peculiar type of behaviour in groups called serpentine behaviour.

\subsection{Serpentine behaviour}

Crowd as an entity is dominated by uniform moods and feelings (Canetti, 1984). In mass religious processions, individuals in a crowd try to move forward deliberately towards their goal. This deliberate movement towards achieving their goal results in emergence of some kind of pattern or structure. Majority of the people attending such processions come in groups consisting of family members, friends, and longtime affiliated members. Group behaviour dominates such religious processions. Kumbh Mela has been notoriously known for families losing their loved ones in large crowd. Hence group members stay together. Two kinds of group behaviour were observed: Groups subdivide into smaller subgroups/free forming groups but as a whole remain in proximity to each other (very regular group behaviour as described in literature); group size varying between 3-8 pedestrians, consisting of very close members (such as women and children) remain in very close proximity and rarely break away from their groups. Members of group stay close to each other with physical contact (by holding hands) and behave as a single large unit of various shapes like serpentine chain, clusters with vulnerables in the middle. These groups try to stay together and do not allow others to penetrate through and thus restricting the differential movement of others in crowd. Therefore this increases the crowd risk by clogging and thereby reducing the efficiency at the exit.

However, it is practically not possible for the group members in the procession to form loops, circles, walk in horizontal direction or any other type of group patterns as the density is very high and all the members in the crowd try to overtake each other. In such cases, during overtaking, one member leads the group and others follow as shown in fig. 5. This Serpentine Behaviour is a special case of group behaviour. The attractive force between leader-follower and repulsive force from other pedestrians lead to formation of flexible chain of member trying to percolate through the crowd. When the distance between the people in the crowd is more, attractive forces dominates. As the distance decreases, repulsive forces are created. This phenomenon ensures that group stays together. During serpentine movement, members hold hands or place their hands on shoulder and do not allow others to penetrate through it. As more and 
more groups continue to form serpentine chains to overtake and move forward, they get compressed against each other with very little breathing space. This behaviour creates an inadvertent body contact with other members in the crowd creating a compressive force on them resulting in pushing. Therefore, presence of large serpentine chain increases the crowd risk.

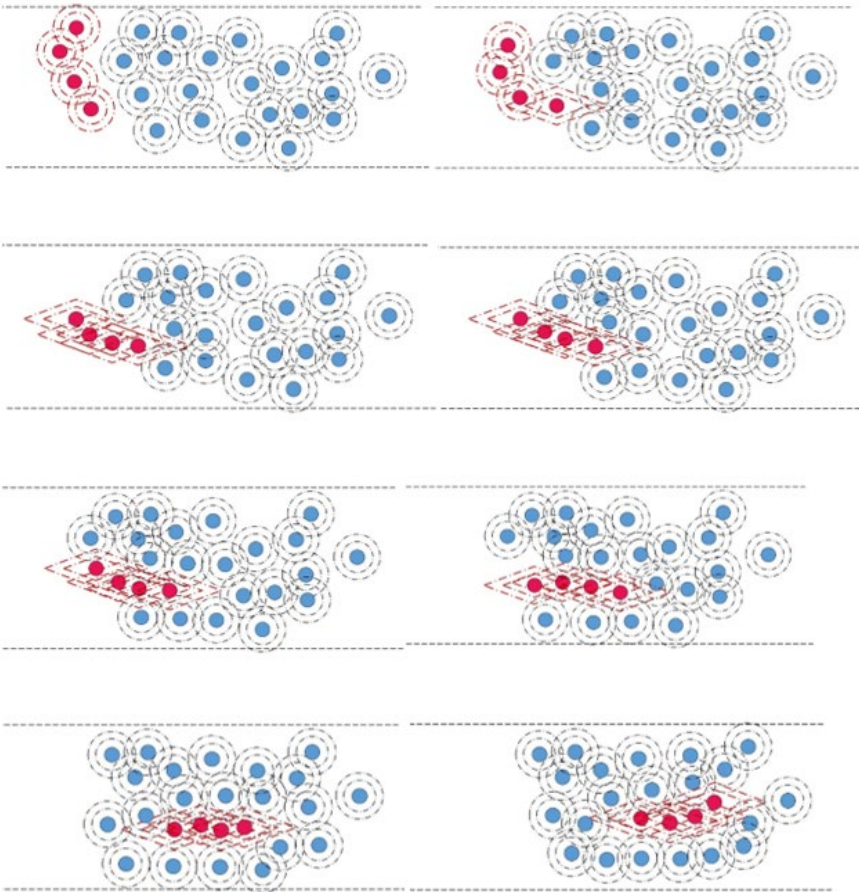

Fig. 5: Serpentine behaviour observed in crowd

\section{Modelling serpentine behaviour}

\subsection{Basic concept of Helbing's social force model}

Helbing's social force model (Helbing and Molnar, 1995) is slightly modified to include the overtaking and serpentine behaviour of pedestrians. Helbing has considered the following four types of forces acting on a pedestrian $i$.

1. Acceleration force towards goal $=\rightarrow_{i}^{0}$

$$
\vec{f}_{i}^{0}=\frac{v_{i}^{0} * \vec{e}_{i}-\vec{v}_{i}}{\tau_{i}}
$$

where,

$v_{i}^{0}$ and $\vec{v}_{i}$ are the desired and actual speeds of pedestrian i

$\tau_{i}$ is the relaxation time

$\vec{e}_{i}$ is the desired direction of pedestrian $\mathrm{i}$

$$
\vec{e}_{i}=\frac{\vec{r}_{i}^{k}-\vec{r}_{l}(t)}{\left\|\vec{r}_{i}^{k}-\vec{r}_{l}(t)\right\|}
$$

where, $\vec{r}_{i}^{k}$ is $\vec{r}_{l}(t)$ are the desired position and actual position of pedestrian i at time $t$.

2. Repulsive force from other pedestrian $\mathrm{j}=\vec{f}_{i j}$

$$
\vec{f}_{i j}\left(\vec{r}_{i j}\right)=-\nabla_{\vec{r}_{i j}} V_{i j}\left[d\left(\vec{r}_{i j}\right)\right]
$$

This force is a monotonically decreasing function of $\mathrm{d}$ (private sphere within which if other pedestrians enter, repulsive effect is created), where,

$\vec{r}_{i j}$ is the position of $\mathrm{i}$ with respect to $\mathrm{j}$ and is calculated as $\vec{r}_{i j}=\vec{r}_{i}-\vec{r}_{j}$ 
$V_{i j}$ is the repulsive potential

3. Repulsive force from obstacle $B=\vec{f}_{i B}$

$$
\vec{f}_{i B}\left(\vec{r}_{i B}\right)=-\nabla_{\vec{r}_{i B}} U_{i B}\left[\left\|\vec{r}_{i B}\right\|\right]
$$

where, $\vec{r}_{i B}$ is the position of $i$ with respect to boundary.

4. Attractive force from obstacles/pedestrians $\alpha=\vec{f}_{i \alpha}$

(Ignored in this study for simplicity)

Therefore, resultant force $\vec{f}_{i}=\vec{f}_{i}^{0}+\vec{f}_{i j}+\vec{f}_{i B}+\vec{f}_{i \alpha}$

\subsection{Forces acting on pedestrians while overtaking}

The following are the three types of forces acting on a pedestrian $\mathrm{i}$ when he tries to overtake $\mathrm{j}$ (refer fig. $6)$.

1. Acceleration force towards goal $=\vec{f}_{i}^{0}$

2. Repulsive force while overtaking pedestrian $\mathrm{j}$ (aligned with $\mathrm{ij}$ ) $=\vec{f}_{i o j}$

3. Repulsive force from obstacle $B=\vec{f}_{i B}$

Boundary B

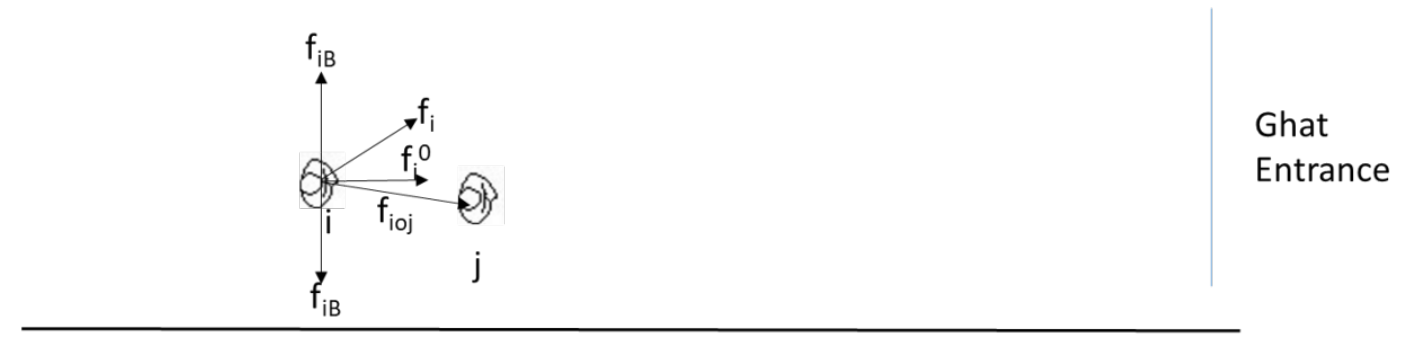

Boundary B

Fig. 6: Forces acting on pedestrians while overtaking

For overtaking, within the private sphere of pedestrian $\mathrm{j}$, speed of $\mathrm{i}$ should be greater than speed of $\mathrm{j}$

$$
v_{i}>v_{j}
$$

Pedestrian may try to overtake either on left or right side of pedestrian $\mathrm{j}$ (Tamura et al 2012)

where,

$$
\begin{aligned}
& r_{\delta L}=r_{j}+d_{\delta 1} e_{i}+d_{\delta 2} e_{\frac{1}{i}} \\
& r_{\delta R}=r_{j}+d_{\delta 1} e_{i}-d_{\delta 2} e_{\frac{1}{i}}
\end{aligned}
$$

$e_{i}$ and $e_{\underline{1}}$ are the desired direction and unit normal to desired direction

$d_{\delta 1}$ and $\stackrel{i}{d}_{\delta 2}$ are longitudinal and lateral distance between pedestrian $\mathrm{j}$ and the next desired position of $\mathrm{i}$

$$
e_{i}(\mathrm{t})=\text { desired direction }=\frac{\vec{r}_{\delta L}^{k}-\vec{r}_{l}(t)}{\left\|\vec{r}_{\delta L}^{k}-\vec{r}_{l}(t)\right\|} \text { or } \frac{\vec{r}_{\delta R}^{k}-\vec{r}_{l}(t)}{\left\|\vec{r}_{\delta R}^{k}-\vec{r}_{l}(t)\right\|}
$$

Based on the position, repulsive force $\vec{f}_{i o j}$ is calculated using equation (2)

Other force calculations would remain the same as social force model.

$$
\text { Resultant force } \vec{f}_{i}=\vec{f}_{i}^{0}+\vec{f}_{i o j}+\vec{f}_{i B}
$$

\subsection{Forces acting on pedestrians in serpentine chain}

The following are the four types of forces acting on pedestrians in serpentine chain (refer fig. 7) 
1. Acceleration force towards goal $=\vec{f}_{i}^{0}$

2. Repulsive force from other pedestrian $\mathrm{j}=\vec{f}_{i j}$

3. Repulsive force from obstacle $B=\vec{f}_{i B}$

4. Attractive force from other pedestrian within group when there are $\mathrm{n}$ number of members in a group $=$ $\vec{f}_{i_{2} i_{1}}, \vec{f}_{i_{3} i_{2}} \ldots . \vec{f}_{i_{n} i_{n-1}}$

Within the serpentine group, the first member leads the group whereas the other members follow their previous member. This following behaviour is introduced as their sub goals with position $\gamma$, which is set behind their respective follower. The sub goal is the attractive force exerted on other pedestrians within the chain. If the number of members in chain is $n$, then there are $m$ sub goals where $n-1=m$ for simplicity. As seen in fig 7, the first member, also known as the leader, will have $\vec{f}_{i j}$ or $\vec{f}_{i o j}$ whereas the followers in the chain will have $\vec{f}_{i_{2} i_{1}}, \vec{f}_{i_{3} i_{2}}, \vec{f}_{i_{4} i_{3}}, \vec{f}_{i_{5} i_{4}}$ and $\vec{f}_{i_{6} i_{5}}$ respectively.

Boundary B

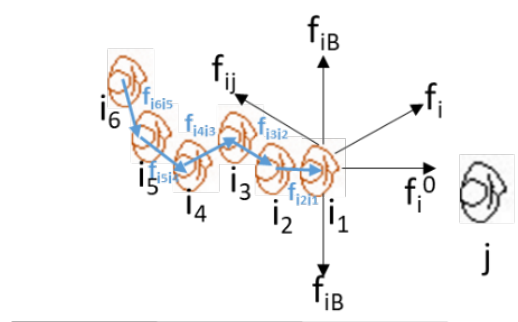

Ghat

Entrance

\section{Boundary B}

Fig. 7: Forces acting on pedestrians in serpentine chain

Position of $\gamma_{1}=r_{\gamma_{1}}=r_{i_{1}}-d_{i_{1} \gamma_{1}} e_{i_{1}}$

Therefore, in general, position of $\gamma_{m}=r_{\gamma_{m}}=r_{i_{n}}-d_{i_{n} \gamma_{m}} e_{i_{n}}$

$$
\begin{aligned}
\vec{f}_{i_{2} i_{1}}^{\gamma_{1}} & =\frac{v_{i_{2}}^{\gamma^{1}} * \vec{e}_{i_{2}}-\vec{v}_{i_{2}}}{\tau_{i_{2}}} \\
\vec{f}_{i_{n} i_{n-1}}^{\gamma_{m}} & =\frac{v_{n}^{\gamma_{m}} * \vec{e}_{i_{n}}-\vec{v}_{i_{n}}}{\tau_{i_{n}}}
\end{aligned}
$$

where,

$\vec{f}_{i_{n} i_{n-1}}^{\gamma_{m}}$ is the attractive force from pedestrian $i_{n-1}$ to $i_{n}$ with $i_{n}$ 's sub goal position $\gamma_{m}$ in the serpentine chain

$v_{n}^{\gamma_{m}}$ and $\vec{v}_{i_{n}}$ is the desired and actual velocity of $i_{n}$ with $i_{n}$ 's sub goal position $\gamma_{m}$ in the serpentine chain

$\vec{e}_{i_{n}}$ is the desired direction of pedestrian $i_{n}$ in the serpentine chain

$\tau_{i_{n}}$ is the relaxation time of $i_{n}$ in the serpentine chain

Repulsive force from obstacles $\vec{f}_{i B}$ is common for all pedestrians in the serpentine chain and remains the same as social force model.

Resultant force $\vec{f}_{i_{1}}=\vec{f}_{i}^{0}+\vec{f}_{i j}+\vec{f}_{i B} \quad\left(i_{1}\right.$ - the first member in the chain)

In case of overtaking, $\vec{f}_{i_{1}}=\vec{f}_{i}^{0}+\vec{f}_{i o j}+\vec{f}_{i B}$ 
Resultant force for the other members (followers) in the chain $\vec{f}_{i_{n}}={\underset{f}{f}}_{i}^{0}+{\underset{f}{i_{n} i_{n-1}}}_{\gamma_{m}}^{\gamma_{i B}}+\vec{f}_{i B}$

\section{Recommendations}

The following are some of the recommendations/suggestions for the event planners and organisers for better management of crowd to avoid circumstances leading to crowd crush and other crowd risk situations.

1. The acceptable level of crowd density should be clearly defined.

2. All Akharas should inform the authorities about the expected number of people likely to participate in the procession from their side.

3. Keeping the above numbers in consideration the flow should be managed in such a way that there is no chaotic movement. (For example, in 2016 Kumbh Mela, near the ghat entrance, the saints were allowed first and the pilgrims were barred from entering. This led to an unstable equilibrium).

4. A proper briefing on the route, ascetic order that the participants need to follow, and the order in which the camps join the procession (ash-smeared sadhus, saints in tractor-drawn chariots, pilgrims) should be provided to participants by the organizers well before the start of the processions.

5. Procession routes should be free from soft boundaries or temporary barricades as they can lead to dangerous situations. The pilgrims should be kept moving to the maximum possible extent by rerouting or by any other strategies, so that the flow is not stopped. This can help in avoiding potential situations leading to crowd crush.

6. The organizers must ensure that the procession starts without any delay. Delayed start is the reason why people started behaving aggressively and tried to push each other to exit their respective camps. Proper information should be provided to people if any delay is unavoidable.

7. Organizers should allow only akhara members to participate in the processions. One suggestion is to issue tickets or badges to the participants so that others do not try to enter the crowd. Others should not be allowed to participate or cross over through the procession.

8. The security personnel should locally control the flow of the procession. Scenarios where people from other camp try to break the flow and overtake should not be allowed with the help of security personnel.

9. The crowd behaviour and its movement should be monitored continuously. Loudspeakers, or any public announcement systems should be used to communicate with the crowd.

10. CCTV monitoring of the crowd along the procession route should be done to identify hazard points and initiating corrective actions.

\section{Summary and Conclusion}

The characteristics of crowd in the procession adds complexities to the dynamics of crowd. Most of the people who participate in the procession come in groups with their affiliated members due to the fear of getting lost or hurt in the crowd. Waiting time due to delay triggers these sleep-deprived people's mind psychologically but still they stay motivated to complete the procession as they consider this as a rare opportunity and feel like they are privileged to be with their saints. This waiting time induces people to exhibit competitive behaviour. Due to this, some people try to percolate through the crowd holding hands of their group members and forming a chain thus exhibiting serpentine behaviour. This causes pressure building in the surroundings and triggers a complex avoidance maneuver in the crowd. As one person starts overtaking, others try to act in the same manner thereby breaking the organized flow.

Therefore, the sense of individuality is almost removed in the crowd. This herding behaviour develops an unstable flow pattern. In addition, the speed of people trying to overtake from the sides is higher than the people walking along the procession. The interaction between people who walk faster and 
slower than the average speed creates an unstable equilibrium in the crowd. All these behaviours lead to situations of crowd risks and suitable measures have to be taken so as to avoid these crowd risks leading to crowd disasters. To model the forces exerted on pedestrians forming serpentine chain, Helbing's social force model is slightly changed to the context of this study.

Many studies have been done to understand pedestrian crowd dynamics and most of them are limited to kinematics of crowd. However, crowd dynamics is a highly complex system. High-density crowds have an enormous potential for overcrowding which could lead to dangerous situations. This paper attempted to highlight the crowd characteristics in high-density event, which have not been captured so far but have a significant impact on crowd behaviour associated with risk. A better understanding of these crowd characteristics will help to increase the efficiency and mitigate the risks in mass events.

Specific recommendations on how to dissipate the forces exerted due to overtaking and serpentine behaviour require detailed research. In addition, only the social forces acting on the pedestrians in the serpentine chain have been considered in this study. Psychological forces, which are predominant in such religious procession, also have a huge impact on crowd and is worth further research.

\section{Acknowledgments}

The work reported in this paper is part of the project titled "The Kumbh Mela Experiment: Measuring and Understanding the Dynamics of Mankind's largest crowd," funded by the Ministry of Electronics and IT Ministry of Communication and Information Technology, Government of India (MITO-0105), Netherlands Organization for Scientific Research, NWO (Project no. 629.002.202), and Robert Bosch Center for Cyber Physical Systems, Indian Institute of Science, Bangalore. (Grant No. RBCO001). The authors also express their gratitude towards Kumbh Mela administration and government of Madhya Pradesh, India for providing constant support and official permissions to carry out research work and establish Indo-Dutch collaboration research camp at Kumbh Mela 2016.

\section{References}

1. "The worst stampede was in Allahabad in 1954, killing 800". The Guardian, 28 August 2003

2. "Varanasi: Deadly stampede at Hindu procession", CNN International Edition, 16 October 2016

3. Canetti, E, "Crowds and Power", Farrar, Straus and Giroux, New York, NY, USA, 1984

4. Cheng L, Yarlagadda R, Fookes C, Yarlagadda P (2014) "A review of pedestrian group dynamics and methodologies in modelling pedestrian group behaviours" World Journal of Mechanical Engineering Vol. 1(1). pp. 002-013.

5. Coleman J S \& James J (1961) "The equilibrium size distribution of freely-forming groups" Sociometry, vol.24, pp-36-45.

6. Helbing D and Molnar P(1995) "Social Force Model for Pedestrian Dynamics," Physical Review $E$, vol. 51, no5, pp.4282-4286

7. Darwin C (1871) "The Descent of Man and Selection in Relation to Sex" John Murray, London 1st edition, http://darwin-online.org.uk/

8. Dave R K (2014) "Crowd Management and Public Safety in India-A new policy initiative", ICT2014, Org 1476577876.

9. Fruin J J (1993) "The Causes and Prevention of Crowd Disasters" First International Conference on Engineering for Crowd Safety, London, England.

10. Gayathri H, Aparna P M, Verma A (2017) "A review of studies on understanding crowd dynamics in the context of crowd safety in mass religious gatherings", International Journal of Disaster Risk Reduction Volume 25, pp 82-91

11. Helbing D, Johansson A, Al-Abideen HE Habib Z (2007) "Crowd turbulence: the physics of crowd disasters" The Fifth International Conference on Nonlinear Mechanics (ICNM-V), Shanghai. 
12. Koster G, Seitz. M, Treml. F, Hartmann. D, Klein. W (2011) "On modeling the influence of group formations in a crowd", Contemporary Social Science 6, 397-414.

13. Kretz T (2007), "Pedestrian Traffic Simulation and Experiments" PhD thesis, Universität Duisburg-Essen

14. Ma J, Song W G, Lo S M and Fang Z M (2013) "New insights into turbulent pedestrian movement pattern in crowd-quakes", Journal of Statistical Mechanics: Theory and Experiment, Volume 2013.

15. Miguel A F (2015) "Key Mechanisms behind Pedestrian Dynamics: Individual and Collective Patterns of Motion" Diffusion Foundations, Vol. 3, pp. 153-164.

16. Moussaid M, Helbing D, Theraulaz G (2011) "How simple rules determine pedestrian behaviour and crowd disasters" Proceedings of the National Academy of Sciences of the United States of America, vol. 108, no. 17, 6884-6888

17. Moussaid M, Perozo N, Garnier S, Helbing D, Theraulaz G (2010) "The walking behaviour of pedestrian social groups and its impact on crowd dynamics", PloS one 5 (4). e10047.

18. Pozner, Vladimir (1990) "Parting with Illusions", Atlantic Monthly Press, 324pp.

19. Strogatz S H, Abrams D M, McRobie A, Eckhardt B, Ott E (2005) "Crowd synchrony on the Millennium Bridge", Nature 438, pp. 43-44, http://dx.doi.org/10.1038/438043a

20. Sindhu K (2014), "Multi-Agent Management of Crowds to Avoid Stampedes in Long Queues", MS thesis, Center for Data Engineering, International Institute of Information Technology, Hyderabad

21. Tamura Y, Phuoc Dai Le, Hitomi K, Chandrasiri N P, Bando T, Yamashita A and Asama H (2012) "Development of Pedestrian Behaviour Model Taking Account of Intention," 2012 IEEE/RSJ International Conference on Intelligent Robots and system (IROS), pp. 482-387 